\title{
Highly Mechanical Performance of Laminated Veneer Lumber Induced by High Voltage Electrostatic Field
}

\author{
Qian $\mathrm{He}^{1}$, Tianyi Zhan ${ }^{1}$, Zehui Ju${ }^{1}$, Haiyang Zhang ${ }^{1}$, Lu Hong ${ }^{1}$, Zhiqiang Wang ${ }^{1}$, Nicolas \\ Brosse $^{2}$, and Xiaoning $\mathrm{Lu}^{1 *}$ \\ ${ }^{1}$ College of Materials Science and Engineering, Nanjing Forestry University, Nanjing, P.R. \\ China \\ ${ }^{2}$ University of Lorraine, LERMAB, Faculty of Science and Technology, Vandoeuvre-lès-Nancy, \\ France. \\ "Corresponding author's e-mail: luxiaoning-nfu@ 126.com
}

\begin{abstract}
The high voltage electrostatic field (HVEF), as a novel technology, was applied in the study to obtain a highly mechanical performance of LVL (laminated veneer lumber) by increasing limiting value of shear failure strength directly affected by bonding strength. The surface property of wood, polymerization extent of PF, bonding interface of wood-to-PF and mechanical properties of LVL were investigated under the HVEF treatment. The results showed that increased free radicals and total surface energy were acquired under the HVEF treatment resulting from more polar groups $(-\mathrm{OH},-\mathrm{CHO})$ and ions were triggered leading to decreased contact angles identified both for Poplar and Masson specimens. The HVEF provided more reactions among wood-to-UF and more cross linking reaction of PF occurred in the treating step. The tendency of vertical density profile was more extremely steep than the control with max density increased by $24.93 \%$ and $30.24 \%$ for Poplar and Masson LVL respectively since adhesive aggregated continuously and orderly along bonding interface and permeation depth reduced to around $200 \mu \mathrm{m}$, accounting for improved bonding shear strength, which eventually brought an enhancement on mechanical properties of LVL with horizon shear strength ( $\|$ and $\perp$ ), modulus of elasticity and static bending strength significantly enhanced by $14.65 \%, 10.68 \%$, $20.67 \%$ and $12.34 \%$ for Poplar LVL and that of Masson LVL enhanced by $17.30 \%, 13.93 \%$, $18.55 \%$ and $12.72 \%$. Besides, the delamination ratio was decreased by $49.57 \%$ and $58.32 \%$ respectively both for Poplar and Masson specimens.
\end{abstract}

KEYWORDS

High voltage electrostatic field; Mechanical property; Bonding interface; Polar groups

\section{INTRODUCTION}

The laminated veneer lumber (LVL) considered as an alternative engineering wood-based composite has been extensively utilized in exterior and interior due to its homogeneous structure and dimensional stability with veneers parallel to each other (Uysal, 2010). Meanwhile, Wood as a hygroscopic material consisting number of chemical groups were highly dependent on the ambient humidity and temperature and PF adhesive were also affected by many factors including 
processing variables and varied temperature, which inevitably lead to low quality, poor exposure durability, terrible bonding property and mechanical performance of LVL (Mirzaei et al., 2016).

Chemical properties of wood mainly components such as cellulose, hemicellulose and ligin processing amount of polar groups $(-\mathrm{OH}$ and $-\mathrm{CHO})$ play a significant role in fiber-to-fiber adhesion and fiber adhesion to polymers (Zhu et al., 2016). Besides, wood could be also appreciated as a porous material due to the hollow structure of cell lumina, pits lying on fiber cell wall and spaces among polymer molecular chains from macroscopic to microscopic scale(Krogell et al., 2013). Bonding property of LVL as a complex process has been investigated in previous studies which merely focused on improving chemical reaction sites and polar groups of wood substrate without wood inherent porous structure taken into consideration, included methods of plasma treatment, magnetic field treatment and corona discharge treatment (Altgen et al., 2016). It is considered that shear failure strength was one of the most paramount factor involved in the mechanic model, of which the limiting value varied with bonding strength (Tsujino et al., 1999). Obviously, mechanical properties of wood based panels are determined by the quality of bonding interface which is evaluated by chemical reaction between adhesive and wood and permeation of adhesive in wood.

The technology of high voltage electric field (HVEF) has been widely utilized due to unique effects on surface performance and interface of various material (Kemp et al., 2016). When porous, nonuniform and anisotropic material were treated by the electrostatic technology, the degree of polarization of that could be improved, resulting from the generation, distribution and interaction of charge on solid surface, which could modulate morphology, dimensionality and strength (Richman, 2015) (Dzubiella et al., 2003). Moreover, triggered ions at the surface of material were occurred with the HVEF treatment and kinetics characteristic (movement direction and rate) of fluid was significantly changed as well (Ghazian et al., 2014). The physicalchemistry property of complex wood-to-UF interface are expected to be influenced by the treatment of HVEF, which has never been utilized before.

Due to the effect of HVEF on surface property and dominating fluid, varied treating time were selected to investigated surface characteristic structure of wood, polymerization extent of PF and bonding interface of wood-to-UF in the experiment, explaining the highly mechanical performance of LVL fabricated by the HVEF.

\section{MATERIAL AND METHODS Material}

The rotary cut veneer of Masson and Poplar respectively from Guangdong Province and Jiangsu Province of China were used as testing materials. The Masson and Poplar veneers were cut to 40 $\mathrm{cm} \times 40 \mathrm{~cm}$ and a thickness of $2.5 \mathrm{~mm}$ and $1.8 \mathrm{~mm}$ respectively, of which the moisture content was $8 \pm 3 \%$. The adhesive was synthetised in the laboratory with the mole ratio of formaldehyde to phenol fixed as 2 to 1 and the solid content of PF adhesive is $47 \%$. The viscosity of PF adhesive is $359 \mathrm{mPa} \cdot \mathrm{s}$ measured by the rotational viscometer (DV-79+Pro, China).

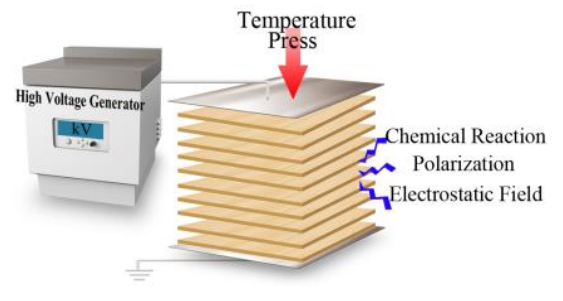


MOC SUMMIT / MAY 2019

Figure 1. The schematic diagram showing the high voltage generator applied in the LVL fabrication process.

\section{LVL PREPARATION}

The manufacturing process of LVL was concisely described in the Figure 1. The adhesive were applied to the single bonding surface approximately $150 \mathrm{~g} / \mathrm{m}^{2}$ and nine veneers were assembled, which were set in the plate electrostatic field within the hot pressing machine. The negative electrode plate connected to the high voltage generator (HC-JDGC-C, China) was assigned on the LVL and the other electrode plate connected to the ground laid under it. Plates were made up of aluminium with the thickness of $3 \mathrm{~mm}$. The high voltage electrostatic field was established in the hot-pressing machine and the value of voltage can be adjusted by rotating the knob on the generator. The electrostatic field intensity was set to $1.5 \mathrm{kV} / \mathrm{mm}$. The temperature was settled at $145^{\circ} \mathrm{C}$. The pressure on the LVL was $1 \mathrm{MPa}$ and the whole time of fabricating specimens was calculated by $60 \mathrm{~s} / \mathrm{mm}$. After completely fabricated by in the HVEF under different treating time, specimen was taken out of the electrostatic field. The repetition for each condition was ten.

\section{MEASUREMENTS}

The free radicals of wood samples were measured by calculated the electron spin resonance (ESR) spectrum (JES-FA200, Japan). Ten samples of each condition treated at temperature of $145^{\circ} \mathrm{C}$ were cut to the dimention of $43 \mathrm{~mm} \times 2 \mathrm{~mm} \times 2 \mathrm{~mm}$, which were dried in the oven at the temperature of $103^{\circ} \mathrm{C}$ for 48 to keep absolute dry $(<3 \%)$. The center field was $326.550 \mathrm{mT}$. Sweep width was $5 \mathrm{mT}$ and sweep time was $2 \mathrm{~min}$. The amplitude was 50 and time constant was $0.3 \mathrm{~s}$.

The dynamic contact angle (OCA40Micro, Germany) was tested after samples were treated under different conditions at $145^{\circ} \mathrm{C}$. The samples were cut to the dimension of $10 \mathrm{~mm} \times 10 \mathrm{~mm}$. The droplets of $10 \mu \mathrm{L}$ were dispensed from a $1 \mathrm{~mL}$ syringe every time at specific heights. The total surface energy and its polar component were following tested using polyethylene glycol (PEG), diiodomethane and deionized water.

Slices were cut from the bonding interface by a slip type slicer with the dimension of $5 \mathrm{~mm} \times$ $5 \mathrm{~mm}$ and a thickness of $30 \mu \mathrm{m}$. The fluorescence microscope (OLYMPUS BX51, China) was used in observing the micro-structure of slices with a UV light source.

The delamination ratio were investigated according to EN 14279-2009 and GB/T 20241-2006. The test was repeated for 10 times and the average of peeling rate could be calculated. The horizontal shear strength was measured by the universal mechanical testing machine (Sansi, Inc.) for twenty times under each condition with the dimension of $6 \mathrm{H} \times 40 \mathrm{~mm}(\perp$, perpendicular to bonding line, $\mathrm{H}$ meas the thickness of $\mathrm{LVL}$ ) and $6 \mathrm{H} \times \mathrm{H}$ (Il, parallel to bonding line). The repetition was ten for each condition. The bonding strength was measured by the compression shear method, of which specimens were tested by the universal mechanical testing machine (Sansi, Inc.) according to the ASTM D905-98. Every condition had ten replicates.

The vertical density profiles were measured by an X-ray densitometer (GreCon DAX-5000, GreCon, Germany) with $0.02 \mathrm{~mm}$ resolution. The dimension of specimens was $5 \mathrm{~mm} \times 5 \mathrm{~mm}$. The ambient temperature was $25^{\circ} \mathrm{C}$ and humidity was $60 \%$. 


\section{RESULTS AND DISCUSSION}

\section{CHARACTERISTICS OF WOOD SAMPLES AND PF ADHESIVE}

The effects of the HVEF on wood material was initially investigated by the ESR (electron spin resonance) and dynamic contact angle as displayed in the Figure 2 - Figure 4. From Figure 2, both the free radicals change ratio of Masson and Poplar samples was increased significantly under the treatment of HVEF regardless of treating time which can be attributable to more chapped covalent bonds among the wood surface and they provided the opportunity for certain extent of non-paired electrons jumping from low-energy state to upper state. Besides, the free radicals change ratio of Masson was more than that of Poplar which sourced from more main components and polar groups such as $-\mathrm{OH},-\mathrm{CHO}$ consisting in softwoods.

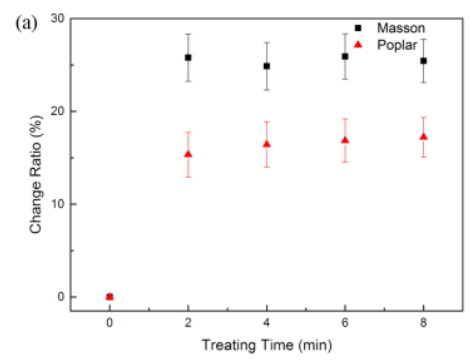

Figure 2. Free radicals with relationship of treating time both for Poplar and Masson samples.

The dynamic contact angle (CA) was also conducted to further identify the property of wood surface. The enhanced surface energy were distinctly showed in the Figure 3 both for the two species with T., D. and P. respectively increased by $17.45 \%, 18.37 \%$ and $68.58 \%$ for Poplar specimens and $21.55 \%, 20.96 \%$ and $59.83 \%$ for Masson specimens which could be explained as more triggered chemical groups and active free ions among wood surface following the HVEF treatment. In hence, decreased contact angles including IA and EA both for Masson and Poplar specimens were also observed in the Figure 3 due to more reactions between chemical groups of PF adhesive and wood surface.
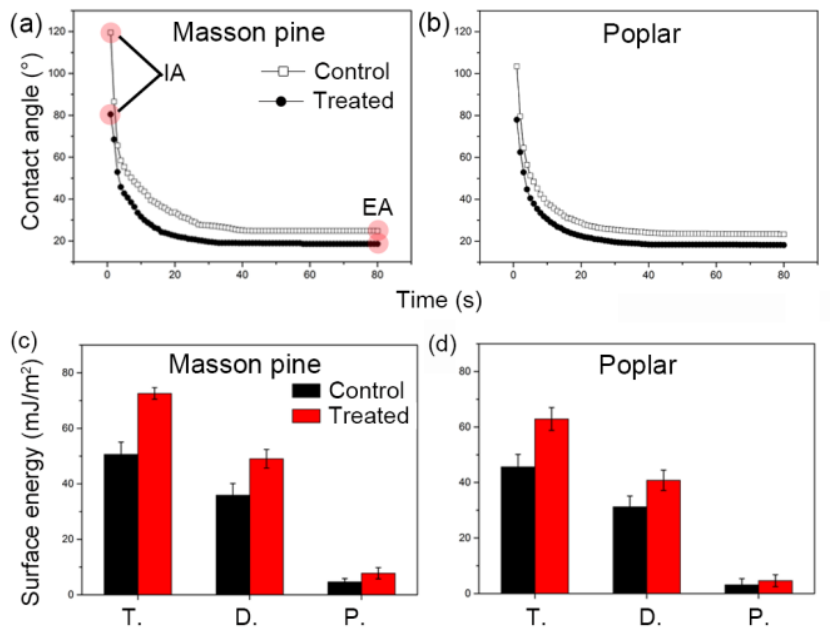

Figure 3. Contact angles and surface energy of the control and treated samples. Noted that $\mathrm{T} .=$ Surface total free energy, P.= Surface free energy of polar components, D.= Surface free energy of dispersion components, IA= Initial contact angle, EA= Equilibrium contact angle. 


\section{THE MICROSTRUCTURE GRAPFS OF LVL SUBJECTED TO THE HVEF}

Increased free radicals and surface energy have significant influence on the bonding performance for wood-based products. The bonding interface of LVL treated by the HVEF for 8 min was discussed as follows with comparison to the control. The fluorescence microscopy was utilized in the measurement to obtain the pattern of bonding interface in the micro scale. The slices were cut from sample via a slip type slicer which were stained by Safranine $\mathrm{T}$ with wood cells presenting as red color and PF adhesive presenting as green color under the UV light in micrographs.

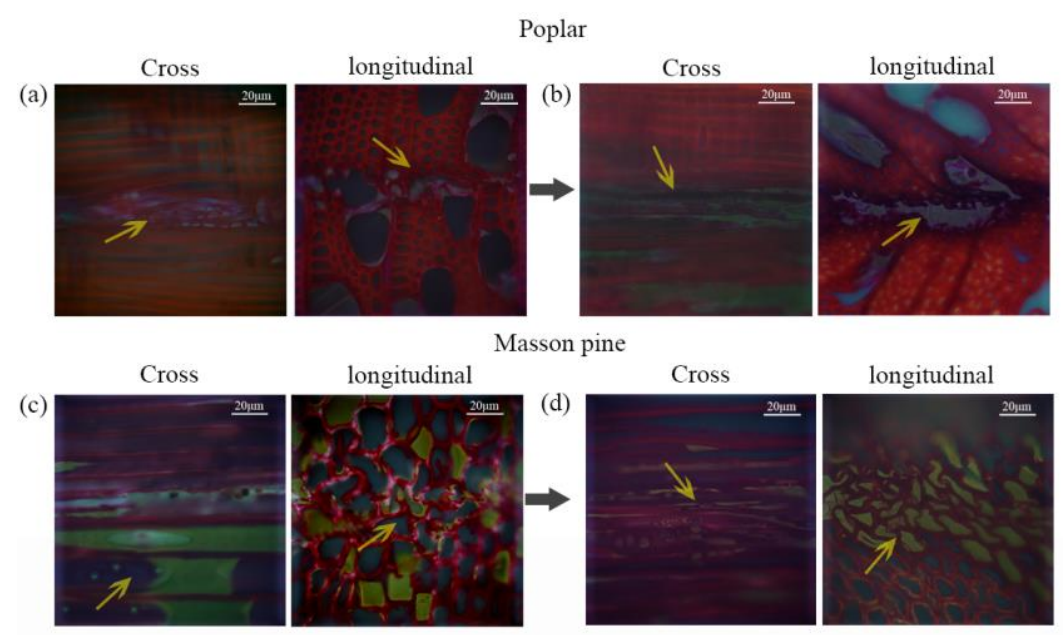

Figure 4. Micrographs of untreated Poplar LVL bonding interface selected from (a) radial section and (b) cross section. Zoomed-in micrographs selected from (c) radial section and (d) cross section.

As depicted in the Figure 4, the distribution of PF adhesive was desultory and irregular among the bonding interface of untreated LVL from radial section and cross section, permeating into the inner of wood veneer via vessels for Poplar, tracheids for Masson and various pits of wood microstructure, donating to the average depth of penetration was about $933 \mu \mathrm{m}$ and $867 \mu \mathrm{m}$ respectively for Poplar and Masson. The micrograph in the Figure 4(a) described that most of adhesive permeated into vessels of Poplar far from the bonding interface leading to few of that involved in the bonding line from both sections. Moreover, as shown in the Figure 4(c), adhesive mostly flowed along the wood cell wall of Masson in the cross section and the quantity of adhesive in lumina of tracheids is little in the radial section. On the contrary, the continuous aggregation behavior of UF adhesive was a remarkable founding with application of the HVEF from the fluorescence micrograph, where the average depth of penetration was around $200 \mu \mathrm{m}$, much less than that of the control. Refer to the Figure 4(b), PF adhesive was orderly filled with vessels of Poplar flowing along the bonding interface and further vessels was empty from both sections. Similarly, the distribution of adhesive was regular and mostly filled with the lumina of tracheids around the bonding interface under the HVEF as displayed in the micrograph of Figure 4(d). It is obviously stemmed from the effect of additional reaction sites and increased polarized ions on wood-to-PF interface as above-mentioned from Figure 2 to Figure 3. 

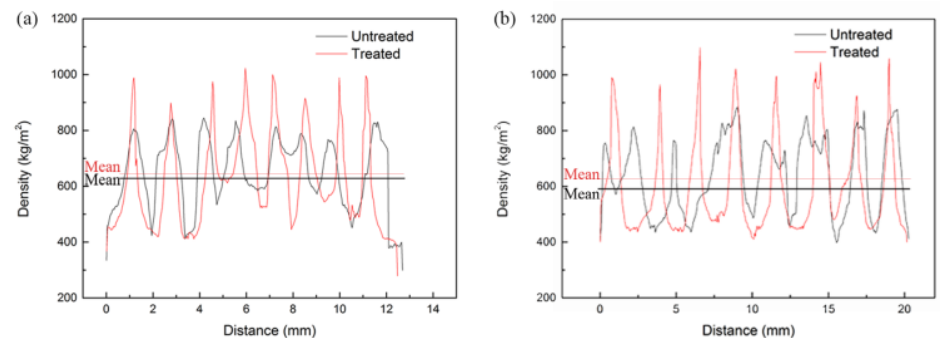

Figure 5. The vertical density profile for(a) Poplar and (b) Masson LVL specimens.

The property bonding interface were also quantized by investigating the vertical density profile in the Figure 5. The tendency of vertical density profile for treated LVL was sharper than that of untreated ones both for Popar and Masson obviously explained by more adhesive aggregated along bonding interface filled with vessels or tracheids and permeation depth of adhesive was much more decreased under the HVEF treatment as shown in the Figure 4. Meantime, the mean value was not significant changed with slightly increased by $4.42 \%$ and $7.32 \%$ for Poplar and Masson respectively but the density of bonding interface performed huge enhancement with increased by $24.93 \%$ for Poplar and $30.24 \%$ for Masson, contributed from more reaction involved in free ions and chemical groups of wood and PF adhesive as explained in the Figure 2 and Figure 3, inevitably leading to the improvement of bonding strength and mechanical properties of LVL.

\section{THE PHYSICAL-CHEMICAL PROPERTY OF LVL SUBJECTED TO THE HVEF}

The delamination ratio was condeucted to recognize the durability of bonding line for treated LVL compared with the control as observed in the Figure 6. The delamination ratio of treated LVL was obviously decreased both for Poplar and Masson with reduced by $49.57 \%$ and $58.32 \%$ respectively under the HVEF treatment. Besides, the bonding strength was measured by compression shear method as displayed in the Table 1. The bonding strength was significantly increased with the treating of HVEF both for Poplar and Masson, which attributed to the more reaction site involved in the wood-to-PF interface and much adhesive aggregating along the bonding line with average value increased by $30.25 \%$ and $43.83 \%$ for Poplar and Masson specimens. The wood failure ratio was observed to be increased as well with average value increased by $41.67 \%$ and $38.46 \%$ for Poplar and Masson specimens.

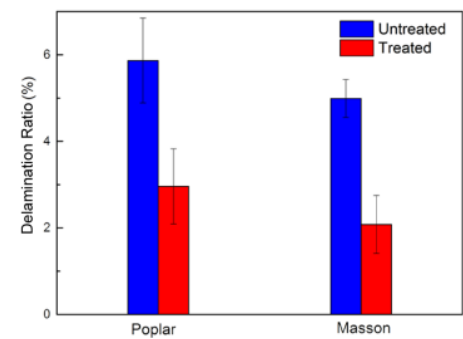

Figure 6. The boiling peeling rate for (a) Poplar and (b) Masson LVL specimens.

Mechanical properties were displayed in the Table 2. The horizon shear strength, modulus of elasticity and static bending strength were measured, which indicated that the increased bonding strength stemming from aggregate adhesive and increased density of bonding line brought an enhancement on mechanical property of LVL both for two species by utilizing the HVEF treatment with the average value of horizon shear strength $(\|$ and $\perp)$, modulus of elasticity and 
static bending strength for Poplar LVL significantly enhanced by $14.65 \%, 10.68 \%, 20.67 \%$ and $12.34 \%$ and that of Masson LVL enhanced by $17.30 \%, 13.93 \%, 18.55 \%$ and $12.72 \%$, which owed to increased limiting value of shear failure strength of mechanical properties highly affected by the bonding property.

Table 1. The bonding strength of untreated and treated LVL. The basic mechanical properties of untreated and treated LVL.

\begin{tabular}{lccc}
\hline Species & Properties & Untreated & Treated \\
& Bonding strength & $6.48(0.50)$ & $8.44(1.12)$ \\
Poplar & $(\mathrm{MPa})$ & & \\
& Wood failure ratio & $60(4.54)$ & $85(4.50)$ \\
& $(\%)$ & & \\
& Bonding strength & $5.43(0.67)$ & $7.81(0.43)$ \\
Masson & $(\mathrm{MPa})$ & & \\
& Wood failure ratio & $65(3.50)$ & $90(4.50)$ \\
& $(\%)$ & & \\
\hline
\end{tabular}

Table 2. The bonding strength of untreated and treated LVL.The basic mechanical properties of untreated and treated LVL.

\begin{tabular}{|c|c|c|c|}
\hline Species & Properties & Untreated & Treated \\
\hline \multirow{4}{*}{ Poplar } & $\begin{array}{l}\text { Horizon shear strength } \\
\qquad(\|, \mathrm{MPa})\end{array}$ & $5.12(0.21)$ & $5.87(0.33)$ \\
\hline & $\begin{array}{l}\text { Horizon shear strength } \\
\qquad(\perp, \mathrm{MPa})\end{array}$ & $4.59(0.24)$ & $5.08(0.29)$ \\
\hline & $\begin{array}{l}\text { Modulus of elasticity } \\
\qquad(\perp, \mathrm{GPa})\end{array}$ & $10.11(0.78)$ & $12.20(0.99)$ \\
\hline & $\begin{array}{l}\text { Static bending strength } \\
\qquad(\perp, \mathrm{MPa})\end{array}$ & $38.16(1.08)$ & $42.87(1.19)$ \\
\hline \multirow[t]{2}{*}{ Masson } & $\begin{array}{l}\text { Horizon shear strength } \\
\qquad(\|, \mathrm{MPa})\end{array}$ & $5.49(0.18)$ & $6.44(0.22)$ \\
\hline & Horizon shear strength & $4.88(0.19)$ & $5.56(0.26)$ \\
\hline
\end{tabular}


MOC SUMMIT / MAY 2019

\author{
$(\perp, \mathrm{MPa})$ \\ Modulus of elasticity \\ $(\perp, \mathrm{GPa})$ \\ $11.43(0.77) \quad 13.55(0.89)$
}

Static bending strength

$(\perp, \mathrm{MPa})$

$43.57(0.82) \quad 49.11(0.88)$

\title{
CONCLUSIONS
}

It was considered that the HVEF treatment has a significant influence on mechanical performance of LVL and other wood-based products by taking most advantage of wood material. Increased free radicals and improved surface characteristic of wood were obtained during treating process, which attributed to more polar groups such as $-\mathrm{OH}$ and $-\mathrm{CHO}$ and free ions triggered by the HVEF. The aggregate adhesive along the bonding interface with permeation depth around $200 \mu \mathrm{m}$ was discovered in micrographs both for Poplar and Masson LVL. Increased bonding strength brought an enhancement on mechanical property of LVL with the average value of horizon shear strength $(\|$ and $\perp)$, modulus of elasticity and static bending strength for Poplar LVL significantly enhanced by $14.65 \%, 10.68 \%, 20.67 \%$ and $12.34 \%$ and that of Masson LVL enhanced by $17.30 \%, 13.93 \%, 18.55 \%$ and $12.72 \%$, which owed to increased limiting value of shear failure strength of mechanical properties highly affected by bonding property. Besides, the delamination ratio was decreased by $49.57 \%$ and $58.32 \%$ respectively both for Poplar and Masson.

\section{ACKNOWLEDGEMENT}

Authors acknowledged funding support by the Study And Demonstration Application Of Green Ecological Bamboo and Wooden Structure System (2017YFC0703500) and the SFA (State Forestry Administration) International Advanced Forestry Science And Technology Introduction Project (2015-4-57). All the authors were contributed equally to this work.

\section{REFERENCES}

Altgen, D., Avramidis, G., Viöl, W., Mai, C. (2016). "The effect of air plasma treatment at atmospheric pressure on thermally modified wood surfaces". Wood Science \& Technology, 50(6), 1-15.

Dzubiella, J., Allen, R.J., Hansen, J.P. (2003). "Electric field-controlled water permeation coupled to ion transport through a nanopore". Journal of Chemical Physics, 120(11), 5001-5004.

Ghazian, O., Adamiak, K., Castle, G.S.P., Higashiyama, Y. (2014). "Oscillation, pseudorotation and coalescence of sessile droplets in a rotating electric field". Colloids \& Surfaces A Physicochemical \& Engineering Aspects, 441(3), 346-353.

Kemp, B.A., Nikolayev, I., Sheppard, C.J. (2016). "Coupled electrostatic and material surface stresses yield anomalous particle interactions and deformation". Journal of Applied Physics, 119(14), 111101-635. 
Krogell, J., Korotkova, E., Eränen, K., Pranovich, A., Salmi, T., Murzin, D., Willför, S. (2013). "Intensification of hemicellulose hot-water extraction from spruce wood in a batch extractor--effects of wood particle size". Bioresource Technology, 143(17), 212-220.

Mirzaei, B., Sinha, A., Nairn, J.A. (2016). "Assessing the role of adhesives in durability of laminated veneer lumber (LVL) by fracture mechanics". Holzforschung, 70(8).

Richman, D.E. (2015). "Conformational responses to changes in the state of ionization of titrable groups in proteins". Holzforschung, 54(6), 604-608.

Tsujino, T., Takeuchi, N., Kawai, T. (1999). "Bending strengths of wood beams of rectangular cross-sections by rigid body spring model, 2: The case where the cause of rupture is shear failure". Journal of the Japan Wood Research Society, 45(1), 9-16.

Uysal, B. (2010). "Effects of the steam test on bonding strength of laminated veneer lumbers manufactured by using different adhesives". Journal of Applied Polymer Science, 99(6), 2973-2977.

Zhu, M., Song, J., Li, T., Gong, A., Wang, Y., Dai, J., Yao, Y., Luo, W., Henderson, D., Hu, L. (2016). "Highly Anisotropic, Highly Transparent Wood Composites". Advanced Materials, 28(26), 5181.

Pennoni, C.R. (1992). "Visioning: The future of civil engineering." Journal of Professional Issues in Engineering Education and Practice, 118(3), 221-233. 